\title{
Alterações do seǵmento posterior na microftalmia: relato de casos
}

\author{
Posterior segment changes in microphthalmic eyes: case report
}

\author{
Fernando José De Novelli ${ }^{1}$ \\ Mário Junqueira Nóbregaª \\ Evandro Luis Rosa ${ }^{3}$ \\ Cristina Martins Faria Bortolotto ${ }^{4}$ \\ Joyce Kuntz ${ }^{5}$
}

\section{RESUMO}

Microftalmia é a alteração de desenvolvimento caracterizada pela redução do tamanho do bulbo ocular. Pode estar associada a manifestações estruturais do segmento anterior e posterior. As alterações mais comuns do segmento anterior são as opacidades corneanas, o estreitamento e fechamento do ângulo da câmara anterior e a catarata, e as mais frequentes no segmento posterior são a efusão uveal, as dobras de retina, a alteração da vascularização capilar macular, a ausência de depressão foveal e a retinosquise periférica. Este estudo descreve o caso de três pacientes com microftalmo e manifestações no segmento posterior documentadas com tomografia de coerência óptica. O primeiro caso apresentou a síndrome de efusão uveal, com descolamento de coróide e de retina, tratada com cirurgia de esclerectomia parcial nos quatro quadrantes. No segundo caso, havia a dobra retiniana neurossensorial na fóvea e feixe papilomacular em ambos os olhos sem o envolvimento de epitélio pigmentado retiniano e coriocapilar. No terceiro paciente, o microftalmo estava acompanhado de ausência da depressão foveal, cujo principal diagnóstico diferencial é a hipoplasia foveal.

Descritores: Microftalmia/patologia; Microftalmia/cirurgia; Microftalmia/complicações; Anormalidades do olho; Doenças da úvea/etiologia, Retina/anormalidades; Fóvea central/anormalidades; Relatos de casos [Tipo de publicação]

Ghanem - Joinville (SC) - Brasil.

${ }^{1}$ Médico Especialista em Retina e Vítreo do Hospital de Olhos Sadalla Amin Ghanem - Joinville (SC) - Brasil.

${ }^{2}$ Doutor, Professor do Curso de Medicina da Universidade da Região de Joinville - UNIVILLE - Joinville (SC) Brasil.

${ }^{3}$ Médico Especialista em Retina e Vítreo do Hospital de Olhos Sadalla Amin Ghanem - Joinville (SC) - Brasil.

${ }^{4}$ Médica Especialista em Oftalmopediatria e Neuroftalmologia do Hospital de Olhos Sadalla Amin Ghanem Joinville (SC) - Brasil.

${ }^{5}$ Residente do Serviço da Pontifícia Universidade Católica - PUC-Curitiba - Curitiba (PR) - Brasil.

Endereço para correspondência: Fernando José De Novelli. Hospital de Olhos Sadalla Amin Ghanem. Rua Carlos Eberhardt, 321 - Joinville (SC) CEP 89218-160 E-mail: fernando.novelli@gmail.com

Recebido para publicação em 29.01.2009

Última versão recebida em 31.03.2009

Aprovação em 20.04.2009

Número de registro clinical trials NCT00811512

Nota Editorial: Depois de concluída a análise do artigo sob sigilo editorial e com a anuência do Dr. João Borges Fortes Filho sobre a divulgação de seu nome como revisor, agradecemos sua participação neste processo.

\section{INTRODUÇÃO}

Microftalmia é a alteração do desenvolvimento caracterizada pela redução do tamanho do bulbo ocular, com medida menor ou igual a $18,5 \mathrm{~mm}$ no indivíduo adulto e $15 \mathrm{~mm}$ na criança ${ }^{(1-3)}$. Essa condição pode ser determinada por herança autossômica dominante ou recessiva, ou surgir esporadicamente.

Apesar do seu menor tamanho estrutural, olhos microftálmicos apresentam, em geral, a dimensão do cristalino preservada, o que leva à possibilidade de estreitamento ou fechamento do ângulo da câmara anterior. Também há relatos de outras alterações do segmento anterior, como opacidades corneanas e catarata.

No segmento posterior, as manifestações decorrentes da microftalmia são mais variadas e incluem efusão uveal, dobras de retina, alteração de vascularização capilar macular, ausência de depressão foveal e retinosquise periférica. A alteração estrutural da esclera, associada ao seu espessamento, requer atenção, especialmente em situações cirúrgicas, devido ao risco do aparecimento da síndrome de efusão uveal ${ }^{(4)}$.

O objetivo deste estudo é descrever três casos de microftalmia com manifestações características no segmento posterior, documentadas com tomografia de coerência óptica.
} 


\section{RELATO DE CASOS}

\section{Caso 1}

Paciente do sexo feminino de 39 anos, branca, relatava história de diminuição visual gradual em ambos olhos (AO) há três meses. Apresentava AV corrigida de 20/200 no OD e conta dedos a dois metros no OE. A refração era de $+18,00 \mathrm{DE}$ em AO. A biomicroscopia evidenciava câmara anterior rasa e cristalino transparente em AO, pressão intraocular 16 mmHg em ambos olhos. A fundoscopia mostrava descolamento total de retina associado a descolamento de coróide periférico em 360 graus em AO. A ultrassonografia ocular revelou comprimento axial bilateral de $16 \mathrm{~mm}$ e, à tomografia de coerência óptica, observaram-se dobras de retina com hiporrefletividade subretiniana correspondendo ao descolamento de retina neurossensorial (Figura 1A).

A paciente foi submetida a cirurgia de esclerectomia parcial nos quatro quadrantes em $\mathrm{AO}$, com o objetivo de promover a drenagem passiva do líquido acumulado nos espaços supracoróideo e sub-retiniano. Apesar de ocorrer melhora anatômica e funcional inicial, houve recidiva do descolamento de coróide e retina. Procedeu-se nova cirurgia, com aplicação de mitomicina nos sítios previamente abordados, o que levou à resolução do descolamento e à estabilização da acuidade visual em 20/400 em AO, nos 18 meses subsequentes.

\section{Caso 2}

Paciente do sexo feminino de 31 anos, branca, referia baixa visual desde a infância. Apresentava AV corrigida de 20/60 em $\mathrm{AO}$ e refração de $+16,00 \mathrm{DE}$ em AO. A biomicroscopia evidenciava câmara anterior rasa e cristalino transparente em AO, pressão intraocular $15 \mathrm{mmHg}$ AO. A fundoscopia mostrava ausência da depressão foveal e distúrbio pigmentado na meia periferia em ambos olhos.

A ultrassonografia mostrou comprimento axial de $16,5 \mathrm{~mm}$ no OD e 16,6 mm no OE. A tomografia de coerência óptica revelou ausência de depressão foveal bilateral. Não se observaram sinais de líquido sub ou intrarretiniano em AO (Figura $1 \mathrm{~B}$ referente à retinografia do $\mathrm{OD})$.

\section{Caso 3}

Paciente do sexo masculino de 26 anos, branco, relatava baixa visual desde a infância. Apresentava AV corrigida de 20/80 em AO e refração de +17,00 DE em AO. A biomicroscopia mostrava câmara anterior profunda e cristalino transparente em $\mathrm{AO}$ e a pressão intraocular era $12 \mathrm{mmHg}$ em AO. A fundoscopia evidenciava dobra retiniana horizontal na fóvea e no
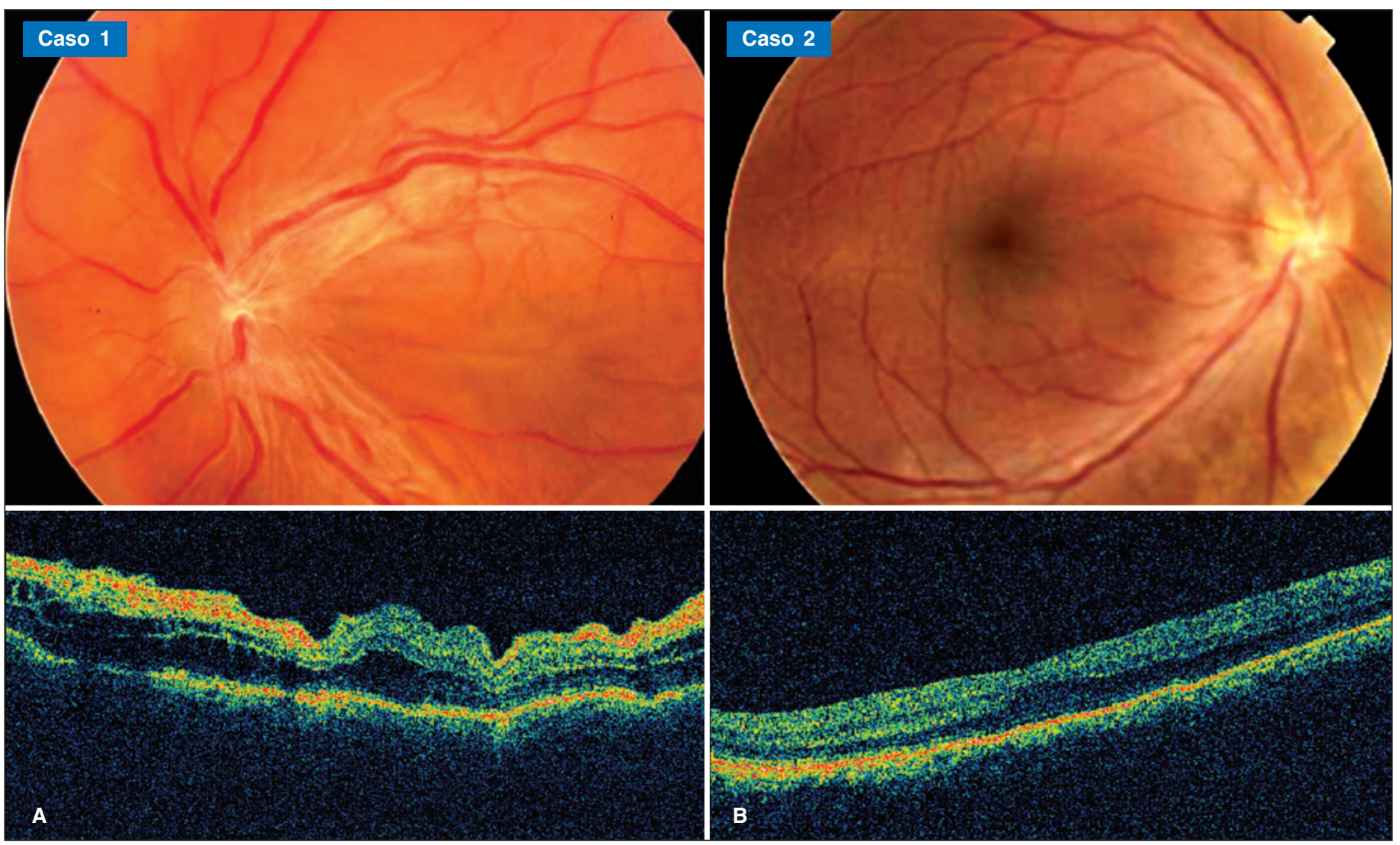

Figura 1 - A) OCT referente ao OE do caso 1 (retinografia): descolamento de retina neurossensorial presente na efusão uveal; B) OCT referente ao OD do caso 2 (retinografia): evidenciando ausência de depressão foveal e retina aplicada e mácula lisa, alteração do epitélio pigmentar na região de média periferia 
feixe papilomacular em AO. A ultrassonografia mostrou comprimento axial de $15,4 \mathrm{~mm}$ no OD e $15,3 \mathrm{~mm}$ no OE. A tomografia de coerência óptica revelou, na região da dobra retiniana, a presença de pequenos espaços císticos hiporreflexivos na retina interna em AO. Não havia envolvimento do complexo epitélio pigmentado retiniano-coriocapilar na dobra (Figura 2).

\section{DISCUSSÃO}

O primeiro caso mostrou o microftalmo associado à síndrome de efusão uveal idiopática. Trata-se de uma rara condição, inicialmente descrita por Schepens e Brockhust ${ }^{(5)}$ em 1963, caracterizada por um descolamento de coróide e retina, secundário ao vazamento de líquido da coriocapilar para o espaço supracoróideo e sub-retiniano ${ }^{(6)}$. Geralmente é bilateral e afeta indivíduos de meia idade portadores de microftalmo.

Nos casos crônicos de efusão uveal, a alteração do epitélio pigmentado da retina é evidente e denominada "manchas de leopardo". Acredita-se que o espessamento escleral observado nestes casos tenha importante papel na fisiopatologia do descolamento de coróide e retina. E, com o objetivo de promover o escoamento do líquido supracoróideo e sub-reti- niano para o espaço peribulbar posterior, preconiza-se o tratamento cirúrgico através da esclerectomia parcial nos 4 quadrantes.

Houve recidiva do quadro após a primeira operação. Para evitar a adesão da cápsula de Tenon à esclera e aumentar a drenagem passiva do líquido, realizou-se nova intervenção, em que se aplicou mitomicina $0,25 \mathrm{mg} / \mathrm{ml}$ durante dois minutos no leito escleral previamente ressecado. Apesar de a acuidade visual não melhorar significativamente após a segunda cirurgia, observou-se bom resultado anatômico uma vez que, num seguimento pós-operatório de 18 meses, não houve recorrência da efusão uveal.

No segundo caso, foi observado o microftalmo associado à ausência de depressão foveal. A tomografia de coerência óptica mostrou espessamento das camadas retinianas externas na fóvea, devido ao aglomerado de células neurossensoriais, sem a presença de líquido intra ou sub-retiniano.

A ausência de depressão foveal nos pacientes microftálmicos não é infrequente e não determina, por si só, o comprometimento da função visual ${ }^{(7)}$. A hipoplasia foveal também pode mostrar ausência de depressão foveal semelhante à microftalmia. No entanto, além de apresentar o comprimento axial ocular normal, a hipoplasia foveal costuma estar associada a outras alterações tais como intensa diminuição da acuidade
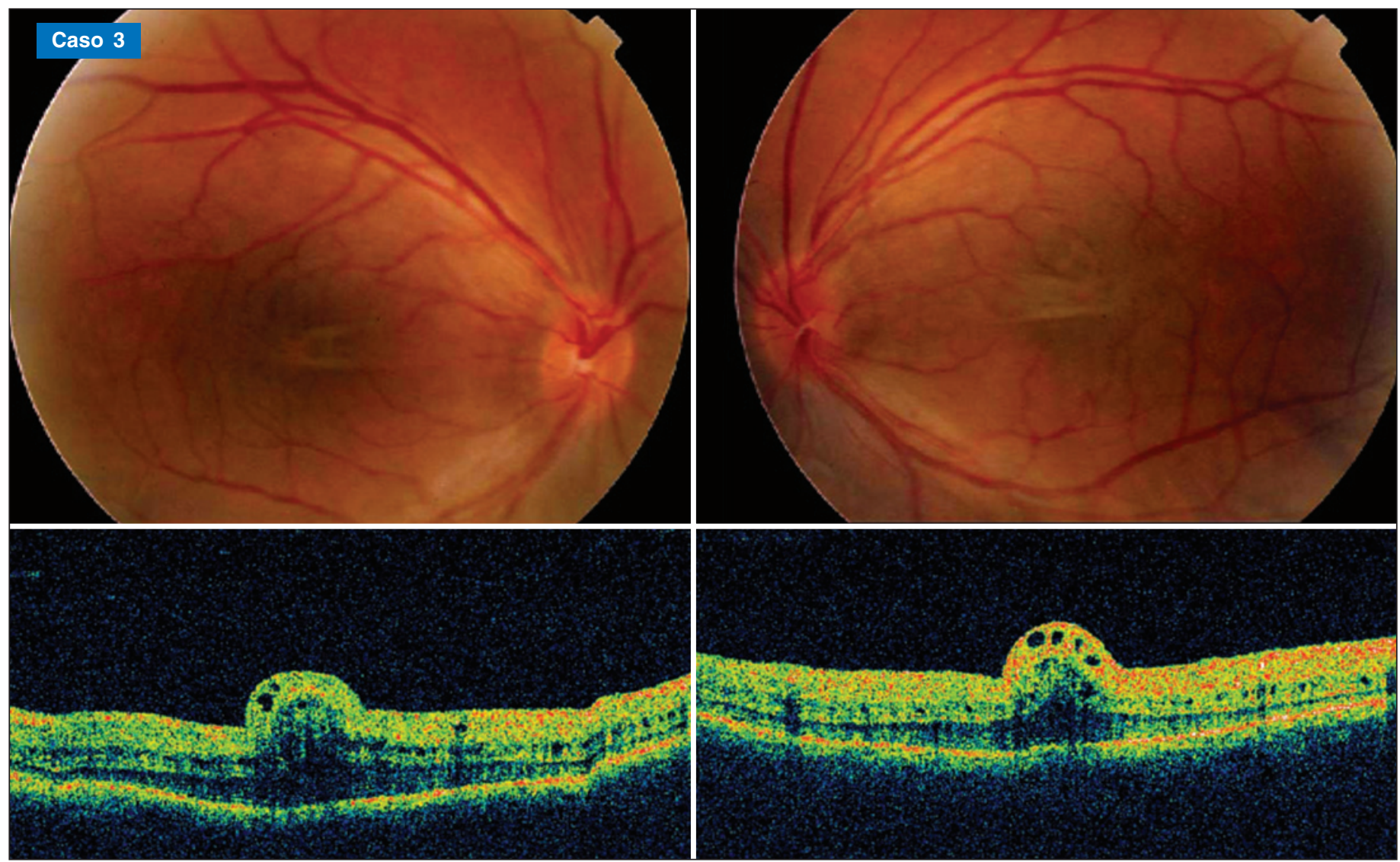

Figura 2 - Caso 3 (OD à esquerda e OE à direita). Retinografia: presença de dobras de retina neurossensorial no feixe papilomacular; OCT: evidencia que o epitélio pigmentar e coriocapilar não participam na formação da dobra retiniana. 
visual, nistagmo, albinismo, retinopatia da prematuridade e acromatopsia.

O terceiro caso apresentou microftalmo posterior. Trata-se de um tipo raro de microftalmia, onde há desproporcionalidade entre o segmento anterior, anatomicamente normal, e o segmento posterior, com alterações fundoscópicas evidentes ${ }^{(8-9)}$. As principais características oculares encontradas no microftalmo posterior são a alta hipermetropia, a efusão uveal, a dobra coriorretiniana, o pseudopapiledema e a ausência ou redução da zona avascular foveal ${ }^{(10)}$.

Nesse caso, além da alta hipermetropia, havia a dobra retiniana neurossensorial na fóvea e feixe papilomacular em ambos os olhos. A tomografia de coerência óptica mostrou a característica peculiar de redundância da retina interna sem a participação do epitélio pigmentado retiniano e coriocapilar. Isso ocorre devido ao crescimento independente da retina neurossensorial e do epitélio pigmentado da retina nos estágios de embriogênese, particularmente na região macular. Por tudo isso, a tomografia de coerência óptica revelou ser o exame de excelência para o diagnóstico desta entidade patológica.

Os três casos relatados mostram que a microftalmia pode estar associada a outras manifestações oculares que levam ao maior comprometimento visual. A ciência dessas eventualidades por parte dos oftalmologistas é fundamental para que se proceda o melhor tratamento e acompanhamento dos pacientes que apresentam esses quadros incomuns.

\section{ABSTRACT}

Microphthalmos is a developmental ocular disorder defined as a small eyeball. The condition can be associated with abnormalities of anterior and posterior segments. The most common anterior characteristics include corneal opacities, angle-closure and a shallow anterior chamber and cataract. The main findings of posterior segment are uveal effusion, retinal folds, abnormalities of macular capillar vascularization, absence of foveal depression and peripheral retinoschisis. Three patients with microphthalmos were assisted and their OCT features of posterior segment were analyzed. The first case had uveal effusion syndrome, choroidal and retinal detachment treated with parcial sclerectomy at the four quadrants. The other case presented with neurosensory retinal fold at fovea and papillomacular area in both eyes without involvement of retinal pigment epithelium layer and choriocapillaris. The third patient showed absence of foveal depression. The main diferencial diagnosis for this condition is foveal hypoplasia.

Keywords: Microphthalmos/pathology; Microphthalmos/surgery; Microphthalmos/complications; Eye abnormalities; Uveal diseases/etiology; Retina/abnormalities; Fovea centralis/abnormalities; Case reports [Publication type]

\section{REFERÊNCIAS}

1. Weiss AH, Kousseff BG, Ross EA, Lonbottom JL. Simple microphthalmos. Arch Ophthalmol. 1989;107(11):1625-30.

2. Van Duyse D. Microphthalmos. In: Duke Elder S, editor. System of Ophthalmology. St. Louis: Mosby; 1983. p.488-95.

3. Warburg M. Classification of microphthalmos and coloboma. J Med Genet. 1993;30(8):664-9.

4. Wu W, Dawson DG, Sugar A, Elner SG, Meyer KA, McKey JB, Moroi SE Cataract surgery in patients with nanophthalmos: results and complications. J Cataract Refract Surg. 2004;30(3):584-90.

5. Gass JD, Jallow S. Idiopathic serous detachment of the choroid, ciliary body and retina (uveal effusion syndrome). Ophthalmology. 1982;89(9):1018-32.

6. Brockhurst RJ. Nanophthalmos with uveal effusion. A new clinical entity. Arch Ophthalmol. 1975;93(12):1289-99.

7. Bijlsma WR, van Schooneveld MJ, Van der Lelij A. Optical coherence tomography findings for nanophthalmic eyes. Retina. 2008;28(7):1002-7.

8. Spitznas M, Gerke E, Bateman VB. Hereditary posterior microphthalmos with papillomacular fold and high hyperopia. Arch Ophthalmol. 1983;101(3):413-7.

9. Khairallah M, Messaoud R, Zaouali S, Ben Yahia S, Ladjimi A, Jenzri S Posterior segment changes associated with posterior microphthalmos. Ophthalmology. 2002;109(3):569-74.

10. Aras C, Ozdamar A, Ustundag C, Ozkan S. Optical coherence tomographic features of papillomacular fold in posterior microphthalmos. Retina. 2005;25(5):665-7. 